\title{
Variação sazonal de espécies de tripes em videira de acordo com sistemas de manejo e fases fenológicas
}

\author{
Andréa Nunes Moreira(1), José Vargas de Oliveira(2), José Eudes de Morais Oliveira( ${ }^{(3)}$, \\ Andréa Costa Oliveira ${ }^{(3)}$ e Ingride Dayane de Souza ${ }^{(3)}$
}

\begin{abstract}
(1)Instituto Federal de Educação, Ciência e Tecnologia do Sertão Pernambucano, Campus Zona Rural, BR 235, Km 22, Projeto Senador Nilo Coelho, N4, Caixa Postal 178, CEP 56300-00 Petrolina, PE. E-mail: andrea.nunes@ifsertao-pe.edu.br (2)Universidade Federal Rural de Pernambuco, Departamento de Agronomia/Entomologia, Rua Dom Manoel de Medeiros, s/no, Dois Irmãos, CEP 52171-900 Recife, PE. E-mail: vargasoliveira@uol.com.br ${ }^{(3)}$ Embrapa Semiárido, BR 428, Km 152, Zona Rural, Caixa Postal 23, CEP $56302-970$ Petrolina, PE. E-mail: jose.eudes@cpatsa.embrapa.br, deiahhh@gmail.com, ingridceecc@yahoo.com.br
\end{abstract}

Resumo - O objetivo deste trabalho foi avaliar a ocorrência de espécies de tripes em videira e a flutuação populacional, das espécies mais frequentes e abundantes, em diferentes fases fenológicas e sistemas de manejo. $\mathrm{O}$ experimento foi realizado em Petrolina, PE, em cinco parreirais - três da cultivar Sugraone (certificada, semiconvencional e convencional) e duas da cultivar Brasil (certificada e convencional). Foram coletadas folhas e inflorescências durante dois anos consecutivos (2008-2010), em 15 plantas. Foram realizadas análises de correlação entre as médias de infestação de tripes e os fatores meteorológicos temperatura, umidade relativa do ar e precipitação pluvial. O número médio de larvas e adultos de tripes foi comparado entre os sistemas de manejo e as fases fenológicas. Nas folhas, foram constatadas as espécies Heliothrips haemorrhoidalis, Retithrips syriacus, Selenothrips rubrocinctus, Frankliniella schultzei, Frankliniella sp. e Scolothrips sp., além de larvas de Aelothripidae. Nas inflorescências, foram identificadas apenas espécies de Thripidae, F. schultzei, F. brevicaulis, F. rodeos, F. gardeniae e Frankliniella sp. Não houve correlação de temperatura, umidade relativa e precipitação pluvial com o número médio de tripes. Existe um complexo de espécies de tripes na videira, e a fenologia da planta exerce influência na abundância desses insetos.

Termos para indexação: Vitis vinifera, abundância, fatores meteorológicos, fenologia, Thysanoptera.

\section{Seasonal variation of thrips species in grapevine according to phenological stages and management systems}

\begin{abstract}
The objective of this work was to evaluate the occurrence of thrips species and the population fluctuation, of the most frequent and abundant ones, in different management systems and phenological stages of grapevine. The experiment was carried out in Petrolina, PE, Brazil, in five vineyards - three with the cultivar Sugraone (certified, semi-conventional, and conventional) and two with the cultivar Brasil (certified and conventional). Leaves and flowers were collected during two subsequent years (2008-2010) from 15 plants. Correlation analyses were performed between the means of thrips infestation and the meteorological variables temperature, relative humidity, and rainfall. The average number of larvae and adult thrips was compared between the management systems and phenological stage. The thrips species found on leaves were: Heliothrips haemorrhoidalis, Retithrips syriacus, Selenothrips rubrocinctus, Frankliniella schultzei, Frankliniella sp., and Scolothrips sp., as well as nymphs of Aelothripidae. On flowers, only the Thripidae species was found: F. schultzei, F. brevicaulis, F. rodeos, F. gardenia, and Frankliniella sp. There was no correlation between temperature, relative air humidity, and rainfall with the average number of thrips. There is a thrips species complex on grapevine, and plant phenology affects the abundance of the insects.
\end{abstract}

Index terms: Vitis vinifera, abundance, meteorological variables, phenology, Thysanoptera.

\section{Introdução}

A vitivinicultura na região semiárida vem se destacando no cenário brasileiro, em virtude da alta produtividade, do volume da produção e da qualidade da uva (Vitis vinifera L.) (Silva et al., 2009).
Em 2011, o Submédio do Vale do São Francisco, especificamente o polo Petrolina, PE e Juazeiro, BA, produziu aproximadamente 250 mil toneladas em uma área de 11.000 ha (Agrianual, 2011), tornando a uva uma das principais frutas brasileiras na pauta de exportação.

Pesq. agropec. bras., Brasília, v.47, n.3, p.328-335, mar. 2012 
$\mathrm{Na}$ região semiárida, os processos fisiológicos da videira são acelerados, em decorrência de fatores climáticos, principalmente temperatura, e de técnicas de produção, sendo possível obter até duas safras e meia por ano, dependendo da cultivar. A data de poda passa a ser a referência para o início do ciclo fenológico, que é definido de acordo com o mercado importador (Silva et al., 2009). As podas são realizadas, geralmente, de dezembro a fevereiro e de maio a julho, com colheitas entre abril e junho e setembro e novembro, respectivamente, para estabelecer e manter uma arquitetura da planta que facilite o manejo, com indução da produção de gemas que originem brotos frutíferos e regulação do crescimento vegetativo (Leão \& Rodrigues, 2009).

A alteração do agroecossistema provocada pela intensidade de manejo e a expansão da área cultivada pode propiciar condições favoráveis ao surgimento de problemas fitossanitários, com destaque para os relacionados à ocorrência de pragas, como os tripes (Haji et al., 2009). Estes insetos têm sido citados como importantes pragas em uvas finas de mesa em diversos países da Europa, América do Sul e Estados Unidos (Lopes et al., 2002; Morse \& Hoddle, 2006; Mujica et al., 2007a). No Brasil, Monteiro (2002) relatou a ocorrência de Frankliniella schultzei (Trybom), Heliothrips haemorrhoidalis (Bouché) e Retithrips syriacus Mayet, todos da família Thripidae. Lopes et al. (2002) observaram a presença de $F$. occidentalis (Pergande) (Thripidae) em área de cultivo da uva 'Niagara', em Limeira, SP. Na região de Marialva, PR, duas espécies de Thripidae, F. rodeos (Moulton) e F. gardeniae Moulton, foram relatadas em uva 'Itália' (Botton et al., 2007). Em Caxias do Sul, RS, as espécies $F$. rodeos e $F$. serrata Moulton (Thripidae) foram consideradas como praga principal da videira por 35,6\% dos produtores (Formolo et al., 2011). No Submédio do Vale do São Francisco, três espécies desta mesma família - Selenothrips rubrocinctus (Giard), R. syriacus e Frankliniella sp. - são responsáveis por perdas qualitativas nas bagas de uvas finas de mesa, principalmente quando se destinam à exportação (Haji et al., 2009).

As espécies $S$. rubrocinctus e $R$. syriacus se alimentam das folhas, o que provoca o aparecimento de manchas amareladas, que, posteriormente, se tornam marrons. Em altas infestações, causam necrose e desfolhamento parcial ou total da planta (Haji et al., 2009). Espécies de Frankliniella provocam, nas bagas, a formação de um halo esbranquiçado, ao redor de uma pequena cicatriz no local de postura (Jensen et al., 1981).

Para realizar o controle de tripes, no contexto do manejo integrado de pragas, o conhecimento das espécies e de suas flutuações populacionais, ao longo do ciclo de desenvolvimento da cultura, são fundamentais.

O objetivo deste trabalho foi avaliar a ocorrência de espécies de tripes e a flutuação populacional, das espécies mais frequentes e abundantes, em diferentes sistemas de manejo e fases fenológicas da videira.

\section{Material e Métodos}

O experimento foi realizado em cinco áreas com videira: três com a cultivar Sugraone (sem semente) e duas com a Brasil (com semente), em Petrolina, PE. Os sistemas de manejo avaliados com a cultivar Sugraone foram: uva certificada (CE), de acordo com os princípios da produção integrada de uvas finas de mesa; uva sem aplicação de inseticidas (SI), que não utiliza o controle químico das pragas; e uva convencional (CO), sem o monitoramento ou a tomada de decisão quanto ao controle ou não das pragas, com a aplicação de inseticidas realizada por calendário. Com a cultivar Brasil, foram avaliados os sistemas de manejo: uva certificada (CE) e convencional (CO). As amostragens de folhas e inflorescências foram realizadas entre 8 e 10 h da manhã, em datas não simultâneas, em razão dos procedimentos de manejo da poda, de acordo com o mercado importador.

A área SI (cultivar Sugraone) situa-se no campo experimental do Instituto Federal de Educação, Ciência e Tecnologia do Sertão Pernambucano, e as áreas CE (cultivares Sugraone e Brasil) e CO (cultivares Sugraone e Brasil) foram selecionadas de plantios comerciais. As características de cada área estão descritas na Tabela 1.

A coleta de folhas foi realizada em intervalos de, aproximadamente, 15 dias, tendo-se coletado, ao acaso, duas folhas das posições apical, mediana e basal dos ramos apicais, medianos e basais de cada planta. As amostras coletadas em 15 plantas foram subdivididas em cinco parcelas de três plantas seguidas, dentro de uma mesma fileira. As folhas foram acondicionadas em sacos de papel, envoltas com sacos de plástico, etiquetadas e levadas ao laboratório. O material foi acondicionado sob refrigeração, e foi realizada a contagem do número de larvas e adultos de 
tripes, conforme Mound \& Kibby (1998), sob estéreo microscópio, com aumento de até 40 vezes. Larvas de tripes encontradas em folhas foram acondicionadas em placas de Petri contendo folhas, para obtenção dos adultos e descrição das injúrias. Os espécimes foram preservados em álcool etílico a 70\%, e larvas e adultos foram enviados para identificação. Exemplares de cada espécie coletada encontram-se depositados no Laboratório de Entomologia da Embrapa Semiárido.

As inflorescências foram amostradas ao se subdividir a área experimental em cinco parcelas de dez plantas, tendo-se coletado uma inflorescência por planta, aos 30 ( $100 \%$ botão floral), 35 ( $40 \%$ flores abertas) e 40 (100\% flores abertas) dias após a poda. As inflorescências foram cortadas com tesoura de raleio, colocadas em saco de papel envolto por saco de plástico e levadas ao laboratório para dissecação e contagem dos tripes. Por ocasião da colheita, cachos de uva foram coletados para observação das injúrias causadas por tripes, que foram comparadas às injúrias relatadas na literatura. Os procedimentos de preservação e identificação do material foram os mesmos que os descritos anteriormente.

Foi realizada análise de correlação linear simples entre os dados médios de infestação de tripes na folha e os valores de temperatura média, umidade relativa do ar e índice pluviométrico. Os dados meteorológicos foram obtidos de estação meteorológica localizada na Empresa Fruitfort, Petrolina, PE $\left(09^{\circ} 22^{\prime} \mathrm{S}\right.$ e $\left.40^{\circ} 33^{\prime} \mathrm{W}\right)$. O número médio de larvas e adultos de tripes (na folha e na inflorescência) foi comparado entre os sistemas de manejo de cada cultivar e as fases fenológicas da videira, por meio de análise de variância e teste de Tukey, a 5\% de probabilidade. Os dados originais foram transformados em raiz $(\mathrm{x}+0,5)$. As fases fenológicas analisadas foram: brotação, crescimento vegetativo, floração, frutificação e amadurecimento, colheita e repouso. As análises foram realizadas com uso do programa SAS, versão 8.02 (SAS Institute, 2001).

Para a análise faunística, foram utilizados os índices de frequência, constância, dominância e abundância dos tripes coletados em folhas, conforme Silveira Neto et al. (1976). As espécies consideradas dominantes foram as mais abundantes e as que obtiveram os maiores índices faunísticos de frequência, constância e dominância.

\section{Resultados e Discussão}

$\mathrm{Na}$ cultivar Sugraone, na área SI, foram registrados, nas folhas, os tripes fitófagos $R$. syriacus, H. haemorrhoidalis, S. rubrocinctus, F. schultzei e Frankliniella sp., enquanto, nas inflorescências, foram verificadas as espécies $F$. rodeos, $F$. gardeniae, F. brevicaulis, F. schultzei e Frankliniella sp. Na mesma cultivar, na área $\mathrm{CE}$, foram constatados apenas os tripes R. syriacus (folha) e Frankliniella sp. (folha e flor). Já na $\mathrm{CO}$, foram encontradas, nas folhas, as espécies, $H$. haemorrhoidalis, $R$. syriacus, S. rubrocinctus e Frankliniella sp. e, nas inflorescências, $F$. brevicaulis e Frankliniella sp.

$\mathrm{Na}$ cultivar Brasil, foram registrados, nas folhas, os tripes H. haemorrhoidalis (CE e CO), R. syriacus (CO), S. rubrocinctus (CE e CO) e, nas inflorescências, F. brevicaulis (CE e CO) e Frankliniella sp. (CO e CE). A espécie predadora Scolothrips sp. foi encontrada na folha em todos os sistemas de manejo, independentemente da cultivar avaliada. Larvas de Aelothripidae foram observadas na cultivar Sugraone (SI e $\mathrm{CO}$ ) e na cultivar Brasil (CO e CE), também nas folhas.

A espécie $R$. syriacus foi a mais numerosa, com 1.167 adultos coletados, e representou $77 \%$ do total de indivíduos, seguida por H. haemorrhoidalis (13\%),

Tabela 1. Características das áreas de videira das cultivares Sugraone e Brasil, em diferentes sistemas de manejo.

\begin{tabular}{|c|c|c|c|c|c|c|c|c|}
\hline Sistema de manejo & № de plantas & Linhas de plantio & Espaçamento (m) & Área $\left(\mathrm{m}^{2}\right)$ & Implantação & Coordenada & s geográficas & Amostragem \\
\hline \multicolumn{9}{|c|}{ Cultivar Sugraone } \\
\hline Certificada & 1.053 & 9 & $3,0 \times 2,0$ & 6.318 & 2002 & $09^{\circ} 17^{\prime} 10,0^{\prime \prime S}$ & $40^{\circ} 25^{\prime} 07,5^{\prime \prime} \mathrm{W}$ & $2 / 6 / 2008$ a $1 / 6 / 2010$ \\
\hline $\begin{array}{l}\text { Sem aplicação } \\
\text { de inseticidas }\end{array}$ & $\begin{array}{c}256 \text { (duas plantas } \\
\text { por cova) }\end{array}$ & 4 & $5,0 \times 3,5$ & 2.240 & 2001 & $09^{\circ} 20^{\prime} 07,2^{\prime \prime S}$ & $40^{\circ} 41^{\prime} 45,1^{\prime \prime} \mathrm{W}$ & $6 / 5 / 2008$ a $2 / 6 / 2010$ \\
\hline Convencional & 768 & 8 & $3,2 \times 2,0$ & $4.915,2$ & 2007 & $09^{\circ} 20^{\prime} 28,6^{\prime \prime S}$ & $40^{\circ} 20^{\prime} 08,0^{\prime \prime} \mathrm{W}$ & $15 / 5 / 2008$ a $1 / 6 / 2010$ \\
\hline \multicolumn{9}{|c|}{ Cultivar Brasil } \\
\hline Certificada & $\begin{array}{c}528 \text { (duas plantas } \\
\text { por cova) }\end{array}$ & 6 & $3,0 \times 3,5$ & 2.772 & 1998 & $09^{\circ} 20^{\prime} 10,0^{\prime \prime S}$ & $40^{\circ} 23^{\prime} 14,0^{\prime \prime} \mathrm{W}$ & $13 / 2 / 2008$ a $6 / 1 / 2010$ \\
\hline Convencional & 1.320 & 8 & $3,2 \times 2,0$ & 8.448 & 2007 & $09^{\circ} 22^{\prime} 54,0^{\prime \prime} \mathrm{S}$ & $40^{\circ} 39^{\prime} 44,6^{\prime \prime} \mathrm{W}$ & $18 / 2 / 2008$ a $3 / 5 / 2010$ \\
\hline
\end{tabular}


F. schultzei + Frankliniella sp. (8\%), Scolothrips sp. $(1,6 \%)$ e $S$. rubrocinctus $(0,4 \%)$, com 197, 121, 25 e 6 indivíduos, respectivamente. Colônias de $H$. haemorrhoidallis e $R$. syriacus foram encontradas na porção abaxial e adaxial das folhas, e o número de adultos na parte superior foi maior, tendo representado 72 e 60,5\%, respectivamente. Foram calculados os índices faunísticos de frequência, abundância, constância e dominância, referentes aos tripes adultos, em folha (Tabela 2). Pelos valores dos índices, $R$. syriacus é a espécie predominante na folhagem da videira.

As espécies $H$. haemorrhoidalis, $R$. syriacus, $F$. schultzei, F. rodeos, F. gardeniae, S. rubrocinctus e Frankliniella sp. já foram relatadas em videira no Brasil (Monteiro et al., 2001; Monteiro, 2002; Botton et al., 2007; Haji et al., 2009). Entretanto, o presente trabalho é o primeiro a reportar $F$. brevicaulis nesta cultura. Para a região do Submédio do Vale do São Francisco, as espécies H. haemorrhoidalis, F. schultzei, F. rodeos e F. gardeniae, os predadores Scolothrips sp. e as larvas de Aelothripidae também foram registrados, pela primeira vez, em associação a uvas finas de mesa. Essa informação é relevante para o manejo integrado de espécies-pragas, pois a sua identificação é uma etapa fundamental.

Foram coletados 14.593 tripes (13.077 larvas e 1.516 adultos) em folhas de videira nos três sistemas de manejo e nas duas cultivares, ao longo dos dois anos de estudo. Nas inflorescências, foram registrados 5.534 tripes (3.387 larvas e 2.147 adultos). Na cultivar Sugraone, na área SI, foi constatada a maior abundância de tripes, que correspondeu ao total de 13.153 tripes (larvas+adultos) na folhagem e de 2.151 tripes (larvas+adultos) na inflorescência. O número de tripes coletados em folhas e inflorescências da cultivar Sugraone, na área CE, foi de 19 e 5, e, na área CO, de 248 e 39, respectivamente, enquanto o da cultivar Brasil foi de 70 e 1.703, na área CE, e de 1.103 e 1.636 na área $\mathrm{CO}$. O número máximo de tripes (257 larvas e 14 adultos) foi observado em uma folha no meio do ramo, na área SI ('Sugraone'). Para a inflorescência, o maior número encontrado de tripes foi de 146, sendo 143 larvas e 3 adultos, também na área SI ('Sugraone'). Chegou-se a observar até 25 adultos em uma única inflorescência.

$\mathrm{O}$ número médio de tripes nas folhas e nas inflorescências, na área SI da cultivar Sugraone, foi superior $(\mathrm{p}<0,001)$ ao das áreas $\mathrm{CE}$ e $\mathrm{CO}$, que não diferiram entre si (Tabela 3). No entanto, na cultivar Brasil, este número foi distinto entre as áreas $\mathrm{CO}$ e CE. A área SI (cultivar Sugraone) foi a que apresentou o maior número de espécies de tripes, quando comparada às demais. Este fato, possivelmente, pode ser atribuído à ausência de aplicações de inseticidas.

A ausência de diferenças entre as densidades médias de tripes, nos diferentes estágios fenológicos, nos sistemas $\mathrm{CE}$ e $\mathrm{CO}$, em ambas as cultivares, indica que, quando o controle destes insetos é realizado, a população tende a permanecer baixa ao longo das fases de desenvolvimento da cultura. Na cultivar Sugraone, na área SI, a brotação e a colheita foram as únicas fases significativamente distintas em relação ao número médio de tripes. No sistema CO, na mesma cultivar, o número observado na floração foi semelhante apenas ao da colheita (Tabela 4).

Resultados similares foram observados por Mujica et al. (2007a), ao avaliar a flutuação populacional de F. occidentalis em uva 'Itália'. Os autores constataram que a maior população de tripes foi encontrada na fase de floração da videira. Já em nectarina, os tripes F. occidentalis, Thrips tabaci Lindeman e F. shultzei foram coletados em maior número próximo à colheita (Mujica et al., 2007b). Desse modo, dependendo da cultura e das espécies de tripes, diferentes estágios fenológicos podem ser infestados por estes insetos.

As injúrias provocadas por $H$. haemorrhoidallis em folhas de videira caracterizam-se por pontuações prateadas ou esbranquiçadas, que se unem em áreas maiores, partindo dos bordos das folhas em direção ao

Tabela 2. Índices faunísticos de adultos de espécies de tripes identificados na folha de videira.

\begin{tabular}{|c|c|c|c|c|c|}
\hline Espécie & № de espécimes & Dominância & Abundância & Frequência & Constância \\
\hline Retithrips syriacus & 1.167 & Dominante & Muito abundante & Muito frequente & Acidental \\
\hline Heliothrips haemorrhoidalis & 197 & Dominante & Dispersa & Muito frequente & Acidental \\
\hline Frankliniella sp. + F. schultzei & 121 & Dominante & Dispersa & Muito frequente & Acidental \\
\hline Selenothrip rubrocinctus & 6 & Não dominante & Rara & Pouco frequente & Acidental \\
\hline Scolothrips sp. & 25 & Não dominante & Rara & Pouco frequente & Acidental \\
\hline
\end{tabular}


centro, geralmente associadas aos excrementos escuros dos insetos (Figura 1 A e B). Essa espécie é nativa da América do Sul (Denmark \& Fasulo, 2010), e os adultos são marrom-escuros com as pernas amareladas (Mound \& Kibby, 1998). Além de ocorrer na videira, também ocorre, no Brasil, em macadâmia, citros, seringueira (Monteiro, 2002) e pinhão-manso (Silva et al., 2008). A espécie também foi relatada na Europa, nos Estados Unidos e na Costa Rica em plantas ornamentais, hortaliças e fruteiras, como mangueira, goiabeira, abacateiro e citros (Mound \& Marullo, 1996; Denmark \& Fasulo, 2010).

As injúrias causadas por $R$. syriacus correspondem às descritas por Haji et al. (2009) e são caracterizadas por manchas cloróticas e, posteriormente, prateadas ou marrons (Figura $1 \mathrm{C}, \mathrm{D}$ e E). Os adultos são de coloração preta, com listras amareladas no dorso, e medem cerca de 1,0 a 1,2 mm de comprimento, e as larvas apresentam coloração avermelhada (Haji et al.,

Tabela 3. Número médio de tripes (larvas+adultos) na folha+inflorescência, em diferentes sistemas de manejo da videira, para as cultivares Sugraone e Brasil ${ }^{(1)}$.

\begin{tabular}{lcc}
\hline Sistema de manejo & № de amostragens & Média \\
\hline Certificada & 51 & 'Sugraone' \\
Sem aplicação de inseticidas & 47 & $0,0015 \pm 0,0007 \mathrm{~b}$ \\
Convencional & 48 & $1,0365 \pm 0,3387 \mathrm{a}$ \\
& \multicolumn{2}{c}{$0,0180 \pm 0,0046 \mathrm{~b}$} \\
Certificada & 43 & 'Brasil' \\
Convencional & 53 & $0,0060 \pm 0,0017 \mathrm{~b}$ \\
\hline
\end{tabular}

${ }^{(1)}$ Médias \pm erro-padrão seguidas de letras iguais, entre sistemas de produção dentro de cada cultivar, não diferem pelo teste de Tukey, a 5\% de probabilidade.
2009). No Brasil, essa espécie também foi encontrada em eucalipto (Monteiro, 2002), sansão-de-campo (Haji et al., 2009), pinhão-manso (Silva et al., 2008) e em plantas das famílias Flacourtiaceae e Polypodiaceae (Pinent et al., 2005). Na Índia e na África do Sul, foi relatada em mamona, mandioca e algodão (Mound \& Kibby, 1998).

A população de $S$. rubrocinctus encontrada foi muito pequena para caracterizar as injúrias em videira. Os adultos dessa espécie apresentam coloração preta ou marrom-escura e medem aproximadamente 1,4 $\mathrm{mm}$ de comprimento. As larvas são amareladas, com os dois primeiros segmentos abdominais vermelhos (Castro et al., 1975). No Brasil, são citados como hospedeiros: cacau, eucalipto, manga (Monteiro, 2002), caju (Castro et al., 1975), lichia (Sánchez-Soto \& Nakano, 2004) e pêssego (Pinent et al., 2008).

Para F. shultzei, observaram-se lesões amarronzadas nos bordos das folhas na fase de brotação, que podem evoluir para murcha dos brotos. Os adultos apresentam coloração geral do corpo marrom, com asas anteriores claras (Monteiro et al., 2001; Barbosa et al., 2005), mas também podem ocorrer formas pretas, que chegam a ser confundidas com as de outras espécies (Chin-Ling et al., 2010). A espécie F. shultzei é amplamente distribuída e pode ser encontrada na Ásia, na África, na Europa, nas áreas do Pacífico, nas Ilhas do Caribe, na América do Sul e nos Estados Unidos (Chin-Ling et al., 2010). No Brasil, tem como hospedeiros: algodão, alface, melão, feijão, rosa, fumo, tomate, melancia, berinjela (Monteiro, 2002), manga (Barbosa et al., 2005), pinhão-manso (Silva et al., 2008), crisântemo e gladíolo (Mound \& Marullo, 1996). No presente

Tabela 4. Número médio de tripes (larvas+adultos) em folhas e inflorescências, em diferentes fases fenológicas e sistemas de manejo de videira, para as cultivares Sugraone e Brasil ${ }^{(1)}$.

\begin{tabular}{|c|c|c|c|c|c|}
\hline \multirow[t]{2}{*}{ Fase fenológica } & \multicolumn{3}{|c|}{ 'Sugraone' } & \multicolumn{2}{|c|}{ 'Brasil' } \\
\hline & Certificada & $\begin{array}{l}\text { Sem aplicação de } \\
\text { inseticidas }\end{array}$ & Convencional & Certificada & Convencional \\
\hline Repouso (folhas) & $0,0014 \pm 0,0006 \mathrm{a}$ & $1,2100 \pm 0,6115 \mathrm{ab}$ & $0,0223 \pm 0,0068 b$ & $0,0098 \pm 0,0059 a$ & $0,1120 \pm 0,1565 a$ \\
\hline Brotação (folhas) & $0,0007 \pm 0,0007 \mathrm{a}$ & $0,0810 \pm 0,0734 \mathrm{~b}$ & $0,0032 \pm 0,0026 b$ & $0,0166 \pm 0,0093 \mathrm{a}$ & $0,1850 \pm 0,1565 \mathrm{a}$ \\
\hline Floração (inflorescência) & $0,0167 \pm 0,0095 \mathrm{a}$ & $5,1590 \pm 2,5518 \mathrm{ab}$ & $0,1300 \pm 0,0409 \mathrm{a}$ & $2,8599 \pm 1,4798 \mathrm{a}$ & $0,1120 \pm 1,2166 \mathrm{a}$ \\
\hline Crescimento vegetativo (folha) & $0,0059 \pm 0,0059 \mathrm{a}$ & $0,4400 \pm 0,1776 \mathrm{ab}$ & $0,0222 \pm 0,0121 b$ & - & $0,0540 \pm 0,1659 \mathrm{a}$ \\
\hline Frutificação e maturação (folha) & $0,0000 \pm 0,0000 \mathrm{a}$ & $1,2570 \pm 0,6318 \mathrm{ab}$ & $0,0049 \pm 0,0018 b$ & $0,0014 \pm 0,0009 a$ & $0,0030 \pm 0,0012 \mathrm{a}$ \\
\hline Colheita (folha) & $0,0000 \pm 0,0000 \mathrm{a}$ & $5,8830 \pm 4,2240 \mathrm{a}$ & $0,0648 \pm 0,0574 \mathrm{ab}$ & $0,0063 \pm 0,0019 a$ & $0,1740 \pm 0,1659 a$ \\
\hline$\overline{\mathrm{F}}$ & 3,32 & 3,46 & 7,25 & 3,76 & 2,58 \\
\hline$\underline{\mathrm{p}}$ & 0,0122 & 0,0099 & 0,0001 & 0,0102 & 0,0368 \\
\hline
\end{tabular}

${ }^{(1)}$ Médias \pm erro-padrão seguidas de letras iguais, na coluna, não diferem pelo teste de Tukey, a 5\% de probabilidade. 
trabalho, a espécie foi constatada nos brotos e nas flores da videira. Segundo Mound (2002), é comum encontrar adultos de duas ou mais espécies de Frankliniella no mesmo conjunto de flores.

As injúrias causadas pelo complexo de tripes das inflorescências foram decorrentes da oviposição, durante o período da floração, o que provocou um halo esbranquiçado nas bagas ao redor de uma pequena cicatriz no local de postura (Figura $1 \mathrm{~F}$ ), conforme observado por Jensen et al. (1981), Botton et al. (2007) e Mujica et al. (2007a). Os insetos da espécie F. brevicaulis apresentam coloração geral do corpo marrom e asas anteriores marrom-claras (Monteiro et al., 2001). A espécie é Neotropical, tendo como

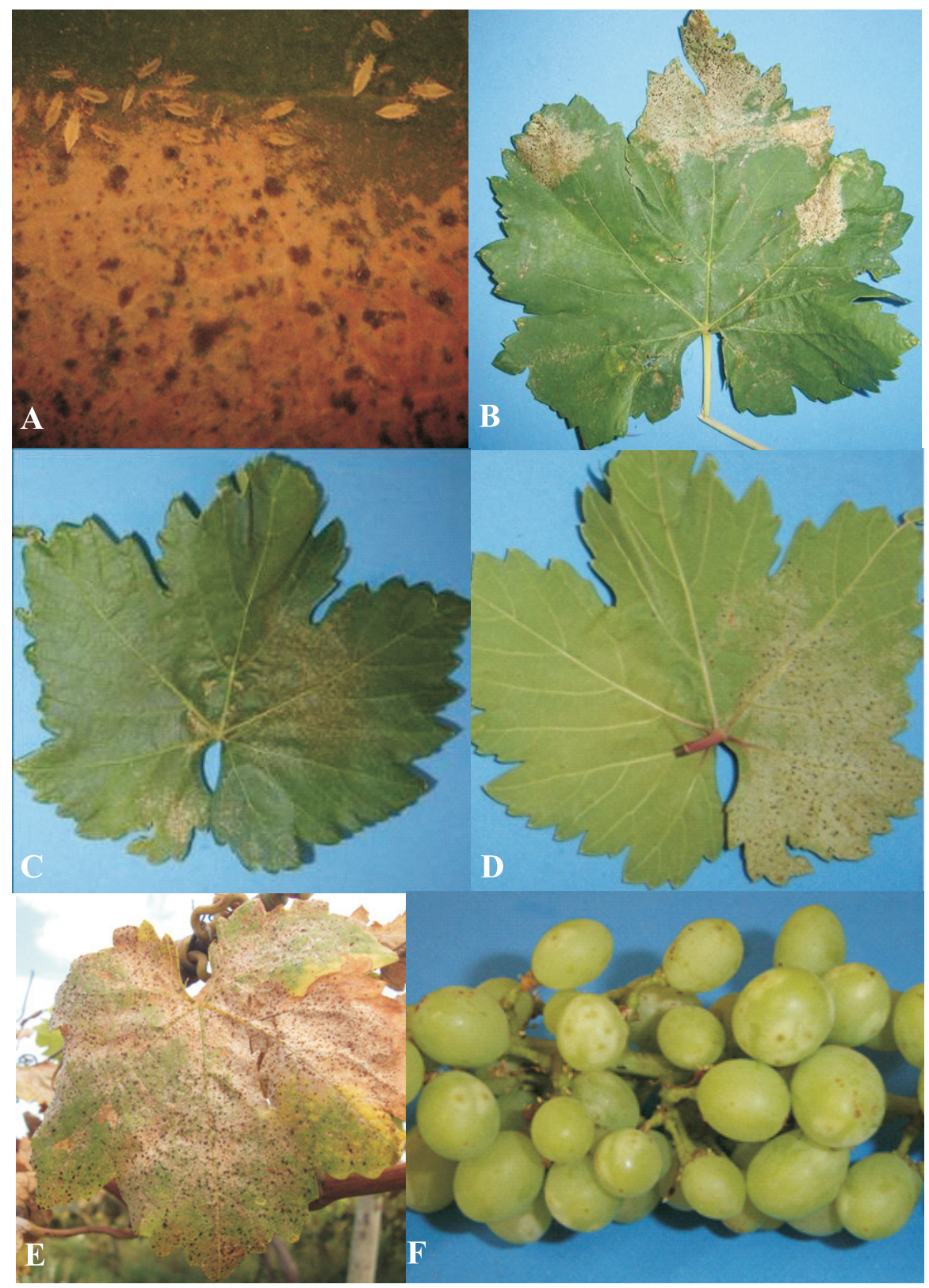

Figura 1. Larvas de Heliothrips haemorrhoidallis (A); injúrias de H. haemorrhoidallis em folhas de videira (B); injúrias de Retithrips syriacus na face superior (C) e na face inferior de folha (D); indivíduos de R. syriacus (E); e injúrias de Frankliniella spp. em baga de videira $(\mathrm{F})$. 
hospedeiros as flores de bananeira, no Brasil (Monteiro, 2002), e as do milho na Costa Rica, no Panamá e em Trindade (Mound \& Marullo, 1996).

Os tripes de $F$. rodeos são de coloração amarela e podem apresentar acentuado polimorfismo em relação ao tamanho e à cor. Esses insetos foram coletados em flores de Asteraceae no Brasil (Cavalleri et al., 2006).

A espécie F. gardeniae, de coloração amarelada, é distribuída em diversos países da América Central (Mound \& Marullo, 1996). No Brasil, foi registrada em flores de gardênia (Cavalleri et al., 2006).

$\mathrm{O}$ fato de terem sido encontrados larvas e adultos de tripes na folhagem e nas inflorescências indica que a videira é um hospedeiro adequado, tanto para a oviposição como para o desenvolvimento das fases larvas dos tripes.

Os tripes do gênero Scolothrips, Tripidae, são bem conhecidos como predadores de ácaros e denominados "tripes de seis manchas", em virtude da presença de seis (raramente cinco) pares de cerdas excepcionalmente longas no pronoto (Mound, 2011). A família Aelothripidae, Terebrantia, apresenta cerca de 250 espécies incluídas em 27 gêneros, das quais poucas ocorrem em regiões tropicais (Mound \& Kibby, 1998). Tradicionalmente, essa família tem sido considerada como a mais primitiva na ordem Thysanoptera, principalmente por causa das asas largas e por alimentarem-se de diversos artrópodes (Mound \& Walker, 1982).

No presente trabalho, não foram observadas correlações significativas entre precipitação, temperatura e umidade relativa com o número de tripes. Entretanto, Lowry et al. (1992) verificaram crescimento populacional em temperaturas mais elevadas.

A abundância de tripes observada no presente trabalho parece ter sido influenciada pela fenologia da videira e pela prática da poda. Esses fatores desempenham importante papel na regulação dos tripes. A poda, por exemplo, reduz diretamente a fonte de alimento com a derrubada das folhas, enquanto a fenologia da planta permite detectar as épocas de maior infestação da praga e, consequentemente, evitar perdas qualitativas na produção da cultura.

\section{Conclusões}

1. Existe um complexo de espécies de tripes nas folhas e nas inflorescências da videira.
2. A espécie Retithrips syriacus é a predominante em folhas de videira.

3. A espécie Frankliniella schultzei está presente em folhas e inflorescências da videira.

4. Os tripes ocorrem nas diferentes fases fenológicas da videira, e a maior infestação é registrada na fase de floração.

5. A temperatura, a umidade do ar e a precipitação não exercem influência sobre a abundância dos tripes em videira, no Submédio do Vale do São Francisco.

\section{Agradecimentos}

À Dra. Renata Monteiro, da Universidade de São Paulo, Escola Superior de Agricultura Luiz de Queiroz, e a Adriano Cavalleri, da Universidade Federal do Rio Grande do Sul, Instituto de Biociências, Departamento de Zoologia, pela identificação dos tripes; e aos produtores Jailson Lira, Ednaldo Aires, Eduardo Campos e Flávio Almeida, por cederam as áreas para a realização deste trabalho.

\section{Referências}

AGRIANUAL: anuário da agricultura brasileira. São Paulo: FNP Consultoria e Comércio, 2011. 482p.

BARBOSA, F.R.; GONÇALVES, M.E. de C.; MOREIRA, W.A.; ALENCAR, J.A. de; SOUZA, E.A. de; SILVA, C.S.B. da; SOUZA, A. de M.; MIRANDA, I. da G. Artrópodes-praga e predadores (Arthropoda) associados à cultura da mangueira no Vale do São Francisco, Nordeste do Brasil. Neotropical Entomology, v.34, p.471-474, 2005

BOTTON, M.; NONDILLO, A.; ZART, M.; PINENT, S.; GENTA, W. Avaliação de inseticidas para o controle de Frankliniella rodeos (Moulton, 1933) (Thysanoptera: Thripidae) em uva de mesa no Brasil. Boletín de Sanidad Vegetal: Plagas, v.33, p.575-580, 2007.

CASTRO, Z.B.; CAVALCANTE, M.L.S.; CAVALCANTE, R.D. Ocorrência de Selenothrips rubrocinctus (Giard, 1901) como praga no Estado do Ceará. Fitossanidade, v.1, p.71-72, 1975.

CAVALLERI, A.; ROMANOWSKI, H.P.; REDAELLI, L.R. Thrips species (Insecta, Thysanoptera) inhabiting plants of the Parque Estadual de Itapuã, Viamão, Rio Grande do Sul state, Brazil. Revista Brasileira de Zoologia, v.23, p.367-374, 2006.

CHIN-LING, W.; FENG-CHYI, L.; YI-CHUNG, C.; HSIEN-TZUNG, S. Species of Frankliniella Trybom(Thysanoptera: Thripidae) from the Asian-Pacific Area. Zoological Studies, v.49, p.824-838, 2010

DENMARK, H.A.; FASULO, T.R. Greenhouse thrips, Heliothrips haemorrhoidalis (Bouche) Insecta: Thysanoptera: Thripidae). Gainesville: University of Florida, 2010. 4p. Available at: <http:// edis.ifas.ufl.edu/pdffiles/IN/IN23200.pdf>. Accessed on: 7 Jan. 2012. 
FORMOLO, R.; RUFFATTO, L.; BOTTON, M.; MACHOTTA JUNIOR, R. Diagnóstico da área cultivada com uva fina de mesa (Vitis vinifera L.) sob cobertura plástica e do manejo de pragas. Revista Brasileira de Fruticultura, v.33, p.103-110, 2011.

HAJI, F.N.P.; OLIVEIRA, J.E. de M.; ALENCAR, J.A. de; GERVÁSIO, R. de C.R.G.; SANTOS, V.F.C. dos; MOREIRA, A.N. Pragas e alternativas de controle. In: SOARES, J.M.; LEAO, P.C. de S. (Ed.). A vitivinicultura no Semiárido brasileiro. Brasília: Embrapa Informação Tecnológica; Petrolina: Embrapa Semi-Árido, 2009. p.515-539.

JENSEN, F.L.; FLAHERTHY, D.I.; LUVISE, D.A. Thrips. In: FLAHERTHY, D.L. (Ed.). Grape pest management. California: University of California, 1981. p.176-186.

LEAO, P.C. de S.; RODRIGUES, B.L. Manejo da copa. In: SOARES, J.M.; LEÃO, P.C. de S. (Ed.). A vitivinicultura no Semiárido brasileiro. Brasília: Embrapa Informação Tecnológica; Petrolina: Embrapa Semi-Árido, 2009. p.295-347.

LOPES, R.B.; TAMAI, M.A.; ALVES, S.B.; SILVEIRA NETO, S.; SALVO, S. de. Occurrence of thrips on Niagara Table grape and its control with the insecticides thiacloprid and methiocarb associated with Metarhizium anisopliae. Revista Brasileira de Fruticultura, v.24, p.269-272, 2002.

LOWRY, V.K.; SMITH, J.W.; MITCHELL, F.L. Life fertility tables for Frankliniella fusca (Hinds) and F. occidentalis (Pergande) (Thysanoptera: Thripidae) on peanut. Annals of the Entomological Society of America, v.85, p.744-754, 1992.

MONTEIRO, R.C. The Thysanoptera fauna of Brazil. In: INTERNATIONAL SYMPOSIUM ON THYSANOPTERA, 7., 2001, Reggio Calabria. Thrips and tospoviruses: proceedings. Canberra: Australian National Insect Collection, 2002. p.325-340.

MONTEIRO, R.C.; MOUND, L.A.; ZUCCHI, R.A. Espécies de Frankliniella (Thysanoptera: Thripidae) de importância agrícola no Brasil. Neotropical Entomology, v.30, p.65-72, 2001.

MORSE, J.G.; HODDLE, M.S. Invasion biology of thrips. Annual Review of Entomology, v.51, p.67-89, 2006.

MOUND, L.A. Species recognition in the genus Scolothrips (Thysanoptera, Thripidae), predators of leaf-feeding mites. Zootaxa, v.2797, p.45-53, 2011.

MOUND, L.A. Thysanoptera biodiversity in the Neotropics. Revista de Biologia Tropical, v.50, p.477-484, 2002.

MOUND, L.A.; KIBBY, G. Thysanoptera: an identification guide. $2^{\text {nd }}$ ed. London: Information Press, 1998. 70p.
MOUND, L.A.; MARULLO, R. The thrips of Central and South America: an introduction (Insecta: Thysanoptera). Gainesville: Associated Publishers, 1996. 487p. (Memoirs on entomology, international, 6).

MOUND, L.A.; WALKER, A.K. Terebrantia (Insecta: Thysanoptera). In: MOUND, L.A. Fauna of New Zealand. Wellington: DSIR, 1982. v.1, p.7-113.

MUJICA, M.V.; SCATONI, I.; FRANCO, J.; NÚÑEZ, S.; BENTANCOURT, C. Fluctuación poblacional de Frankliniella occidentalis (Pergande) (Thysanoptera: Thripidae) en Vitis vinifera L. cv. Italia en la zona sur de Uruguay. Boletín de Sanidad Vegetal: Plagas, v.33, p.457-467, 2007a.

MUJICA, M.V.; SCATONI, I.; FRANCO, J.; NÚÑEZ, S.; BENTANCOURT, C. Fluctuación poblacional de trips (Thysanoptera: Thripidae) en Prunus persica (L.) cv. Fantasía en la zona sur de Uruguay. Agrociencia, v.11, p.39-49, 2007 b.

PINENT, S.M.J.; MASCARO, F.; BOTTON, M.; RED, L.R. Thrips (Thysanoptera: Thripidae, Phlaeothripidae) damaging peach in Paranapanema, São Paulo state, Brazil. Neotropical Entomology, v.37, p.486-488, 2008.

PINENT, S.M.J.; ROMANOWSKI, H.P.; REDAELLI, L.R.; CAVALLERI, A. Thysanoptera: plantas visitadas e hospedeiras no Parque Estadual de Itapuã, Viamão, RS, Brasil. Iheringia. Série Zoologia, v.95, p.9-16, 2005.

SÁNCHEZ-SOTO, S.; NAKANO, O. Primeiro registro de Selenothrips rubrocinctus (Giard) (Thysanoptera: Thripidae) atacando lichia no Brasil. Neotropical Entomology, v.33, p.395-396, 2004.

SAS INSTITUTE. SAS user's guide: statistics version 8 for Windows. Cary: SAS Institute, 2001.

SILVA, P.C.G. da; CORREIA, R.C.; SOARES, J.M. Histórico e importância socioeconômica. In: SOARES, J.M.; LEÃO, P.C. de S. (Ed.). A vitivinicultura no Semiárido brasileiro. Brasília: Embrapa Informação Tecnológica; Petrolina: Embrapa Semi-Árido, 2009. p.21-34.

SILVA, P.H.S. da; CASTRO, M. de J.P. de; ARAÚJO, E.C.A. Tripes (Insecta: Tripidae) associados ao pinhão-manso no Estado do Piauí, Brasil. Revista Brasileira de Oleaginosas e Fibrosas, v.12, p.125-127, 2008.

SILVEIRANETO, S.; NAKANO, O.; BARBIN, D.; VILLANOVA, N.A. Manual de ecologia dos insetos. São Paulo: Agronomica Ceres, 1976. 419p.

$\overline{\text { Recebido em } 6 \text { de abril de } 2011 \text { e aprovado em } 13 \text { de janeiro de } 2012}$ 